\section{Supreme handpiece hygiene}

Synea dental turbines from W\&H combine innovative technology with optimal ergonomics to facilitate high quality treatment.

The lightweight design of these scratchresistant handpieces reduces the risk of strain and fatigue, while LED+ lights ensure shadow-free illumination of the treatment site.

Synea handpieces also feature a patented hygiene head with an antiretraction system to reduce the aerosol being sucked back into the turbine as the rotor slows to a stop.

Additionally, an anti-retraction valve within the turbine itself prevents the backflow of potentially contaminated water via the turbine's waterlines to the central water supply system.

Combat the aerosol challenge today and benefit from exceptional handpiece performance with the Synea range of dental turbines.

To find out more visit www.wh.com/ en_uk, call 01727874990 or email office. uk@wh.com.

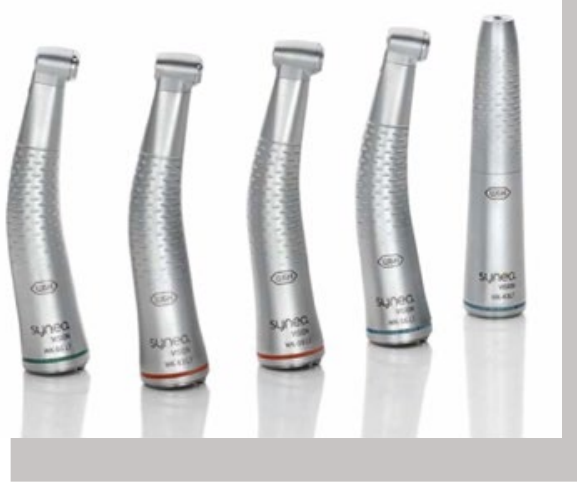

\title{
The simple way to an effective daily clean
}

As we head towards the season of overindulgence, the homecare regimen becomes even more important.

Premium oral hygiene brand, TANDEX, has changed its packaging so patients can easily select the right size brush. Packs now show Passage Hole Diameter (PHD) and ISO numbers - PHD indicates brush size in $\mathrm{mm}$ and ISO is a standard size from 0-8. PHD and ISO together form a unique combination, identified by the colour of the brush handle.

Prevention means better oral health. The simplicity of the TANDEX system gives patients a way to clean gently and effectively.

For more information on Tandex's range of products, visit www.tandex.dk.

\section{World-class teaching and support}

When learning a new skill or broadening your existing capabilities, it is essential that you have access to quality training and long-term support. This is what IAS Academy is all about.

For GDPs looking to expand their skills in the field of ortho-restorative dentistry, IAS Academy offers training courses

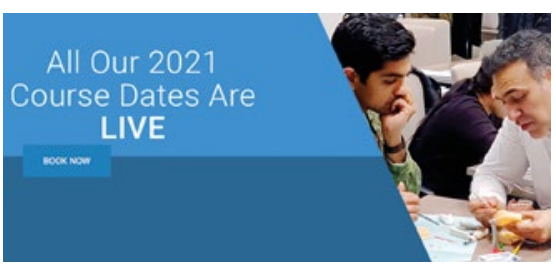

with different entry points to suit various experience levels. Continuous support and mentorship are then readily available from the expert instructors, offering tailored advice and guidance whenever needed to boost confidence and competence.

The IAS Academy membership scheme is also available to provide added benefits like discounts off further training, products and mentoring, as well as extensive resources to support learning.

For more information on IAS Academy membership and upcoming training courses visit www.iasortho.com or call 01932336470 (Press 1).

\section{Floss in the shower}

For a more convenient and effective oral hygiene routine, introduce your patients to the Waterpik Cordless Advanced Water Flosser.

This lightweight, waterproof model can be used in the shower to effectively remove hard to reach bacteria deep between the the gumline. To establish a quick and easy way for your patients to floss, visit www. waterpik.co.uk. teeth and below

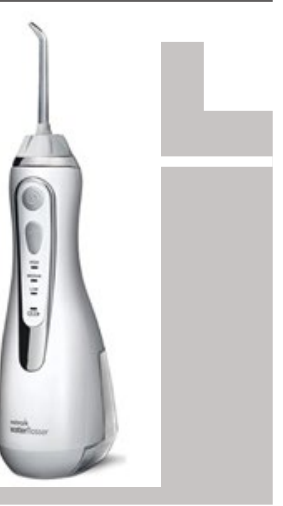

\section{High-performance material}

ClearCorrect is delighted to announce the introduction of a brand-new material for all its clear aligners - ClearQuartz.

The innovative material offers outstanding patient comfort and tooth adaptation in addition to the high, flat trim line, designed to support highly effective, safe and comfortable treatment. It is the latest in tri-layer aligner material technology that caters to the needs and demands of both modern patients and clinicians.

ClearCorrect is part of a comprehensive digital workflow, with various solutions available to ensure an efficient and predictable journey from data capture to aligner manufacturer and treatment monitoring.

For more information on ClearCorrect visit www.straumann.com/clearcorrect/en/ home.html.

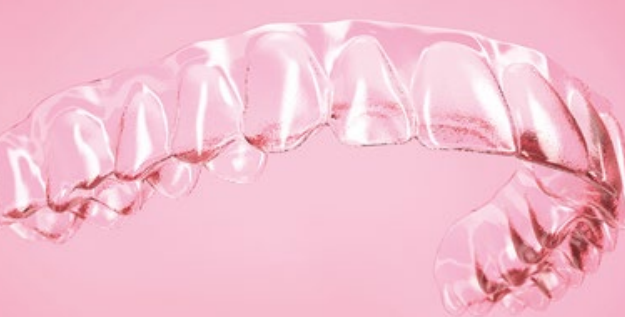

